\title{
Modelling of Facial Soft Tissue Growth for Maxillofacial Surgery Planning Environments
}

\author{
Patrick Vandewalle, Filip Schutyser, \\ Johan Van Cleynenbreugel, and Paul Suetens \\ Medical Image Computing (Radiology - ESAT/PSI), \\ Faculties of Medicine and Engineering, University Hospital Gasthuisberg, \\ Herestraat 49, B-3000 Leuven, Belgium \\ Filip.Schutyser@uz.kuleuven.ac.be
}

\begin{abstract}
When maxillofacial surgery is proposed as a treatment for a patient, the type of osteotomy and its influence on the facial contour is of major interest. To design the optimal surgical plan, 3D image-based planning can be used. However, prediction of soft tissue deformation due to skeletal changes, is rather complex. The soft tissue model needs to incorporate the characteristics of living tissues.

Since surgeon and patient are interested in the expected facial contour some months after surgery when swelling has disappeared, features specific to living tissues need to be modelled. This paper focusses on modelling of tissue growth using finite element methods. This growth is induced by stress resulting from the surgical procedure. We explain why modelling growth is needed and propose a model. We apply this model to 4 patients treated with unilateral mandibular distraction and compare these soft tissue predictions with the postoperative CT image data.
\end{abstract}

Keywords: soft tissue modelling, maxillofacial surgery simulation

\section{Introduction}

The soft tissue model explained in this paper fits into our framework of 3D image-based planning systems. This planning environment adheres to a scenebased approach in which image derived visualizations and additional 3D structures (external to the medical image volume) are co-presented and manipulated. This environment includes tools for osteotomy simulation and distraction simulation [1].

Because of the high impact of distraction therapy on the patient's face, prediction of the soft tissue deformation is highly desirable. Therefore, our planning system also includes a soft tissue model of the skin (i.e. the dermis and the underlying structures like fat and muscles).

Fung [2] reports on the biomechanical properties of living tissues. Skin tissues are called quasi-linear viscoelastic materials, meaning that these tissues show creep, relaxation and hysteresis when applying large oscillations around equilibrium, but the characteristics can be well approximated with linear viscoelasticity 
applying small oscillations. However, this modelling implies demanding computations which are in this application area unrealistic. Moreover, this model describes the biomechanical behavior of soft tissues during a short time interval and not the deformations due to e.g. persistent stress over a longer time.

Different approaches have been investigated to model soft tissues. Teschner et al. describe a multi-layer spring model [3], resulting in short simulation times. However no extended validation study is published. The meshing step, based on the approach of Waters 4], is rather tedious and error-prone. Koch et al. 5], Chabanas et al. 6] and Gladilin et al. [7] use finite element methods to model skin tissue. Koch describes skin tissue as an incompressible elastic tissue. Chabanas adds a muscle activation model to animate the face. Gladilin applies a nonlinear elastic model.

In this paper, we develop a finite element model incorporating growth. In section 2, we explain why it is important to incorporate growth in the model by validating a basic linear elastic model. In subsection 2.3 we explain how we have extended this basic model with a growth component. Simulation results for 4 patients are shown in section 3. After a discussion of these results (section 4), concluding remarks finish this paper (section [5).

\section{Methods}

\subsection{Basic Model}

Our research for an accurate model for maxillofacial surgery planning starts from a linear elastic soft tissue model which is based on the mechanical equilibrium equations

$$
\begin{aligned}
& \frac{\partial \sigma_{x x}}{\partial x}+\frac{\partial \tau_{x y}}{\partial y}+\frac{\partial \tau_{x z}}{\partial z}+F_{x}=0 \\
& \frac{\partial \tau_{x y}}{\partial x}+\frac{\partial \sigma_{y y}}{\partial y}+\frac{\partial \tau_{y z}}{\partial z}+F_{y}=0 \\
& \frac{\partial \tau_{x z}}{\partial x}+\frac{\partial \tau_{y z}}{\partial y}+\frac{\partial \sigma_{z z}}{\partial z}+F_{z}=0
\end{aligned}
$$

with $\sigma_{x x}, \sigma_{y y}, \sigma_{z z}, \tau_{x y}, \tau_{x z}, \tau_{y z}$ the stress components and $\mathbf{F}\left(F_{x}, F_{y}, F_{z}\right)$ the volume forces.

The material properties are introduced into these equations through the constitutive equations relating stresses and strains. The soft tissue is modeled as a homogeneous, linear and elastic material, such that we can use Hooke's law:

$$
\left[\begin{array}{c}
\sigma_{x x} \\
\sigma_{y y} \\
\sigma_{z z} \\
\tau_{x y} \\
\tau_{y z} \\
\tau_{z x}
\end{array}\right]=\frac{E}{(1+\nu)(1-2 \nu)}\left[\begin{array}{cccccc}
1-\nu & \nu & \nu & 0 & 0 & 0 \\
\nu & 1-\nu & \nu & 0 & 0 & 0 \\
\nu & \nu & 1-\nu & 0 & 0 & 0 \\
0 & 0 & 0 & \frac{1-2 \nu}{2} & 0 & 0 \\
0 & 0 & 0 & 0 & \frac{1-2 \nu}{2} & 0 \\
0 & 0 & 0 & 0 & 0 & \frac{1-2 \nu}{2}
\end{array}\right]\left[\begin{array}{c}
\epsilon_{x x} \\
\epsilon_{y y} \\
\epsilon_{z z} \\
\gamma_{x y} \\
\gamma_{y z} \\
\gamma_{z x}
\end{array}\right] \Leftrightarrow \boldsymbol{\sigma}=\mathbf{D} \boldsymbol{\epsilon}
$$


with strain components $\epsilon_{x x}, \epsilon_{y y}, \epsilon_{z z}, \gamma_{x y}, \gamma_{x z}, \gamma_{y z}$, Young's modulus $E$ and Poisson coefficient $\nu$.

If we define $\{\mathbf{X}\}$ as the initial configuration at time $t_{0}$ and $\mathbf{x}=\mathbf{x}(\mathbf{X}, t)$ as the description of the point $\mathbf{X}$ at time $t$, the displacement vector $\mathbf{u}$ can be defined as $\mathbf{x}=\mathbf{X}+\mathbf{u}$. The Green-Lagrange strain tensor relates the strains $\boldsymbol{\epsilon}$ to the displacements $\mathbf{u}: \boldsymbol{\epsilon}=\frac{1}{2}\left(\nabla \mathbf{u}+\nabla \mathbf{u}^{T}+\nabla \mathbf{u}^{T} \nabla \mathbf{u}\right)$. We linearize this equation to $\boldsymbol{\epsilon}=\frac{1}{2}\left(\nabla \mathbf{u}+\nabla \mathbf{u}^{T}\right)$

These equations are discretized using a 3D finite element method. The continuum is modeled as a tetrahedron mesh. For the interpolation between the nodes we use a basic linear, $C_{0}$ continuous shape function using 4 nodes for each tetrahedron [8]. The partial differential equations are reduced to a set of linear equations for the vertices of the tetrahedron mesh: $\mathbf{K} \mathbf{U}=\mathbf{R}$ with $\mathbf{K}=\int_{V} \mathbf{B}^{T} \mathbf{D B} \mathrm{d} V$, $\boldsymbol{\epsilon}=\mathbf{B u}=\left[\begin{array}{llll}\mathbf{B}_{q} & \mathbf{B}_{r} & \mathbf{B}_{s} & \mathbf{B}_{t}\end{array}\right] \mathbf{u}$ and

$$
\mathbf{B}_{m}=\left[\begin{array}{ccc}
\frac{\partial N_{m}}{\partial x} & 0 & 0 \\
0 & \frac{\partial N_{m}}{\partial y} & 0 \\
0 & 0 & \frac{\partial N_{m}}{\partial z} \\
\frac{\partial N_{m}}{\partial y} & \frac{\partial N_{m}}{\partial x} & 0 \\
0 & \frac{\partial N_{m}}{\partial z} & \frac{\partial N_{m}}{\partial y} \\
\frac{\partial N_{m}}{\partial z} & 0 & \frac{\partial N_{m}}{\partial x}
\end{array}\right]
$$

for the 4 tetrahedron vertices $(m=q, r, s, t)$ with shape functions $N_{m}$.

The stiffness submatrix $\mathbf{K}_{m n}^{i}$ is computed for each pair of 2 nodes $m$ and $n$ of an element $i$. It can be computed from the shape function and the material properties as $\mathbf{K}_{m n}^{i}=\mathbf{B}_{m}^{T} \mathbf{D}^{i} \mathbf{B}_{n} V^{i}$ with $V^{i}$ the volume of tetrahedron $i$. The global stiffness matrix $\mathbf{K}$ for the entire model can then easily be assembled from all these element stiffness matrices $\mathbf{K}_{m n}^{i}$.

The derived set of equilibrium equations is constrained by a set of Dirichlet boundary conditions. They force certain displacements to a fixed value. In our facial model there are 2 types of such boundary conditions. First there are the displacements at the border between bone and soft tissue, prescribed by the planned bone displacements. The other boundary conditions are obtained from the assumption that the soft tissue above the eyes and behind the ears will not be affected by the surgery and can thus be supposed to have a fixed position. These Dirichlet boundary conditions can be easily introduced in the equations, as they can all be formulated as $\mathbf{t u}=\mathbf{r}$.

In this first model there are no volume or body forces defined on the tetrahedron mesh. This means that $\mathbf{R}=0$, except for the values introduced by the boundary conditions.

\subsection{Validation}

In the development of a soft tissue model for surgery planning it is very important to be able to see how closely an approximation matches the real postoperative situation. This is the only way to make a good comparison between two implementations and to see if the approximation goals are reached. 


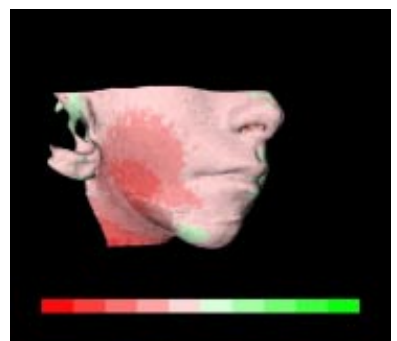

(a)

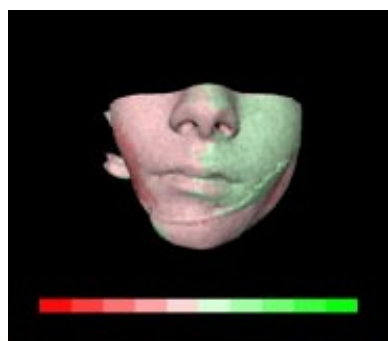

(b)

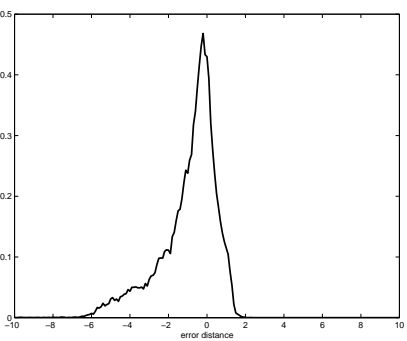

(c)

Fig. 1. (a, b): Color-encoded differences between the real post-operative image and the planned image with the basic model. The color scale covers the interval $[-10 \mathrm{~mm} \ldots 10 \mathrm{~mm}] .(\mathrm{c})$ : Histogram of the distances between the predicted and the real post-operative image.

A procedure also has to be validated with data from different patients. This is needed to make sure that the procedure works not only for one specific patient record, but gives good results for any patient.

\section{Procedure.}

1. 4 months after surgery, the patient gets a CT scan. This post-operative CT scan is rigidly registered to the pre-operative CT data, using maximization of mutual information [9] on an unaltered subvolume as registration method. From these co-registered post-operative data, a surface representation of the skin is generated using the Marching Cubes algorithm [10].

2. Next, a skin surface is created from the pre-operative CT image using the same algorithm.

3. This pre-operative surface is deformed according to the results of our FEM computations. We then have a planned post-operative surface of the skin. This can be compared with the real post-operative surface as they are both registered to the same pre-operative data.

4. For the vertices of this planned post-operative surface the closest distance to the real post-operative surface is computed and visualized using colorcoding. For this coding the normals to the surface are used. They are set to the exterior of the skin surface. When the normal to the real post-operative surface intersects the planned surface in the positive direction, green colors are used for positive values. When it intersects the planned surface in the negative direction, the errors get red colors and negative values.

This results in an easily interpretable image of the errors over the entire surface (figure 1]a,b).

5. In a last step we discard the positional information and an error histogram is made from all the differences (errors) between planned and real postoperative data (figure 1 $\mathrm{c}$ ). Because we would like all errors to be zero, the ideal histogram is a Dirac pulse $\delta$. Therefore the average error $\mu$ and the standard deviation $\sigma$ need to be as small as possible. 


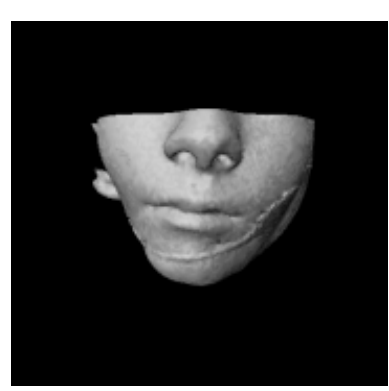

(a)

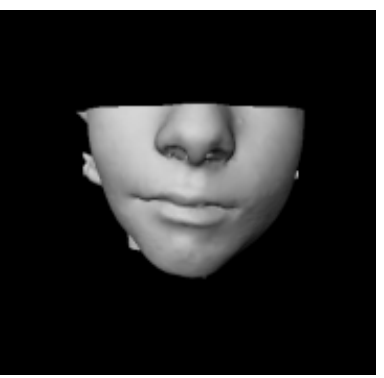

(b)

Fig. 2. Comparison between predicted and real post-operative facial skin envelopes. (a) Predicted post-operative image (The "wrinkle" you notice, is the result of a fixation bandage applied during the acquisition. This should be avoided during the CT examination); (b) Real (registered) post-operative image.

Validation of the Basic Model. We use this procedure to validate the results with the basic model. Figure 2 shows a direct comparison between the predicted (planned) post-operative image and the registered real post-operative image. It is very difficult, even nearly impossible, to make a good comparison between these two images as they are represented here. Therefore, it is more effective to display the differences between the predicted and the real data according to steps 4 and 5 of the validation procedure (see figure 1).

From this histogram we can see that the average error is negative, showing a 'lack of material'. If we analyze the error image (figure 1) and derive positional information (which has disappeared in the histogram), it is clear that mainly in the parts with high stress due to the displacements (close to the bone displacements), large negative errors occur. In these areas the basic model cannot predict reality sufficiently.

\subsection{Soft Tissue Growth}

Model Improvement. When using the basic model, a relatively large residual error is found in the area with large tissue stress (mainly the boundary between displaced and fixed tissue). From Fung [2] and Rodriguez [11] we know that soft tissue will grow under stress conditions. As we try to model the soft tissue envelope about 4 months after surgery, it is very important to include this tissue growth into the model.

As a hypothesis, we state that tissue growth can be modeled as an internal volume force in the tetrahedra, similar to the force caused by a thermal expansion. We replace the previous $\mathbf{R}=0$ by a term

$$
\mathbf{R}=\int_{V} \mathbf{B}^{T} \mathbf{D} \alpha \boldsymbol{\sigma}_{\text {growth }} \mathrm{d} V .
$$

with $\boldsymbol{\sigma}_{\text {growth }}$ the growth stress and $\alpha$ a stability factor (see 2.3). $\boldsymbol{\sigma}_{\text {growth }}$ is a new degree of freedom, which is introduced to fully control the growth. It is a stress induced by the growth process. 


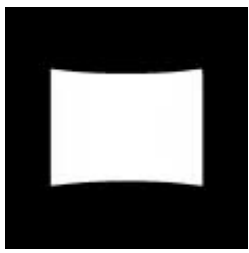

(a) $k=0$

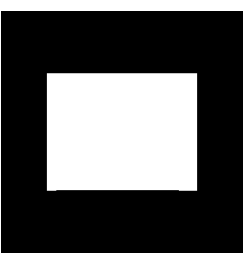

(b) $k=5$

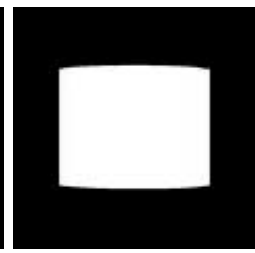

(c) $k=10$

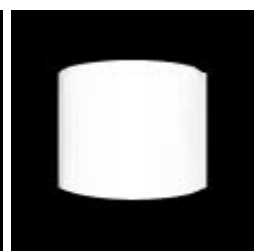

(d) $k=20$

Fig. 3. A cube is stretched to the left and to the right, and the resulting stress in the cube causes growth with different equilibrium states for $k=0,5,10$ and 20 .

The tissue growth is caused by the stress introduced by the bone displacements. Therefore we can state that $\boldsymbol{\sigma}_{\text {growth }}$ is the sum of the stress due to the displacements $\left(\boldsymbol{\sigma}_{d}\right)$ and some extra stress $\boldsymbol{\sigma}_{g}$ generated by the growth process itself (which is induced by the initial displacement stress $\sigma_{d 0}$ ). Therefore we can say $\boldsymbol{\sigma}_{\text {growth }}=\boldsymbol{\sigma}_{d}+\boldsymbol{\sigma}_{g}\left(\boldsymbol{\sigma}_{d 0}\right)$. We assume that $\boldsymbol{\sigma}_{g}=k \boldsymbol{\sigma}_{d 0}$ if $\boldsymbol{\sigma}_{d 0}>0$ and $\boldsymbol{\sigma}_{g}=0$ otherwise. $k$ is the parameter which determines how large the soft tissue growth has to be (figure 3). We then have the following formulation:

$$
\begin{aligned}
\mathbf{R} & =\int_{V} \mathbf{B}^{T} \mathbf{D} \alpha \boldsymbol{\sigma}_{\text {growth }} \mathrm{d} V \\
& =\int_{V} \mathbf{B}^{T} \mathbf{D} \alpha\left(\boldsymbol{\sigma}_{d}+\boldsymbol{\sigma}_{g}\right) \mathrm{d} V \\
& =\int_{V} \mathbf{B}^{T} \mathbf{D} \alpha\left(\boldsymbol{\sigma}_{d}+k \boldsymbol{\sigma}_{d 0}\right) \mathrm{d} V
\end{aligned}
$$

These equations cause the tissue model to grow in an iterative process, which is described in the next paragraph. Iterations are made until an equilibrium state is reached, which happens when $\boldsymbol{\sigma}_{d}=-k \boldsymbol{\sigma}_{d 0}$ if $\boldsymbol{\sigma}_{d 0}>0$ and $\boldsymbol{\sigma}_{d}=0$ otherwise.

Iterative Procedure. To initialize the iterative process, the stresses introduced by the bone displacements are computed. During the first iteration, these stresses are used to compute the 'growth' forces. When the resulting displacements from this first iteration are computed, the remaining stresses are computed again. In the subsequent iterations we always use the stress remaining from the previous iteration to compute $\boldsymbol{\sigma}_{d}$. In this way the residual stress in the soft tissue model is reduced in every iteration until the stresses (and thus also the growth per iteration) is smaller than a certain threshold value.

Stability Factor $\boldsymbol{\alpha}$. In order to keep the iterative growth process controlled and stable, a stability factor $\alpha$ was introduced in the volume force term $\mathbf{R}$. This factor guarantees a safe and stable evolution of the element stresses towards their equilibrium values. $\alpha=0.0005$ is a good value for $\alpha$, as can be derived from figure 4, where the number of iterations needed for convergence is plotted for different values of $\alpha$. For values of $\alpha>0.0006$ the growth process diverges and no stable equilibrium state can be reached any more. 


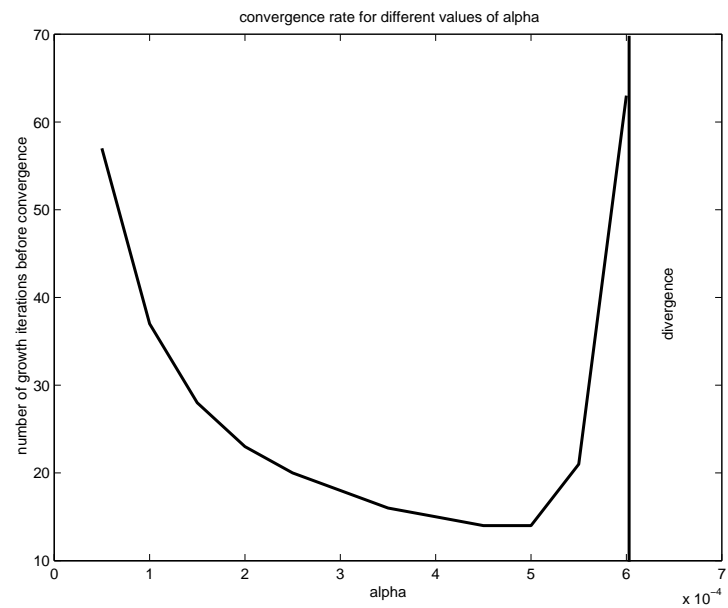

Fig. 4. The number of iterations needed for growth convergence is plotted for different values of $\alpha$. The optimal value (14 iterations in this case) is reached for $\alpha=0.00045$ to 0.0005 .

Parameter Values. The material parameters $E$ and $\nu$ for living soft tissues are hard to measure in practice and they are not described in literature. Young's modulus $E$ is not important for our application, as it appears once in all terms of the equations and can thus be removed from the equations. Contrary to this, the Poisson coefficient $\nu$ has a larger implication on the soft tissue deformation.

Good values for this coefficient $\nu$ and for the growth parameter $k$ can be obtained through an analysis of the error values obtained from the validation on different patient data. The average error and the standard deviation for different values of $\nu$ is shown in figure [5] (a,b), and the same is done for different values of the growth parameter $k$ in figure $5(\mathrm{c}, \mathrm{d})$.

We analyzed the parameter values on the data of 4 different patients and we noted that the optimal values for the different patients are very close to each other. From this we state that these values for the Poisson parameter $\nu$ and the growth parameter $k$ can be used for any arbitrary patient. The parameters $(k$, $\nu)$ are optimal for $(k=20, \nu=0.25)$.

\section{Results}

We illustrate this method for 4 patients suffering from unilateral microsomia. All these patients have been treated with intraoral unilateral mandibular distraction. The distraction device had two degrees of freedom: unidirectional translation and angular rotation.

Using the validation procedure we described earlier, we compare the results obtained with the improved model to the results obtained with the basic model. Predictions resulting from both methods are compared with the real post-operative image. The error distances are shown in figure 6 ] 


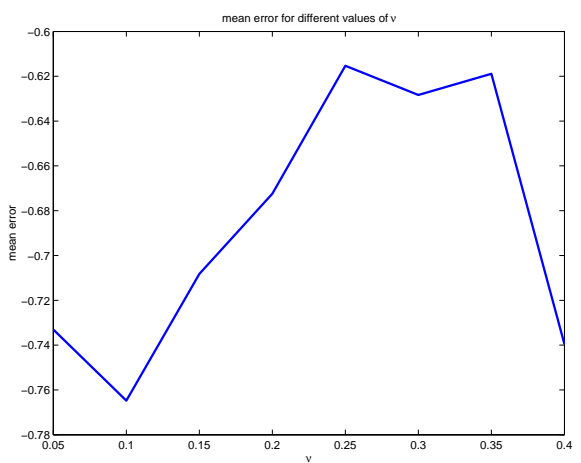

(a) Mean error for different values of $\nu$

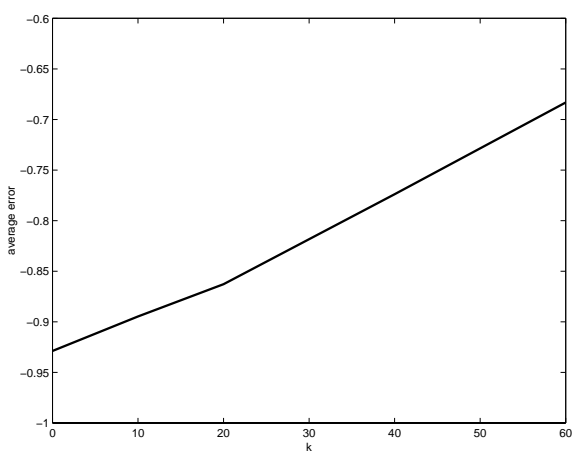

(c) Mean error for different values of $k$

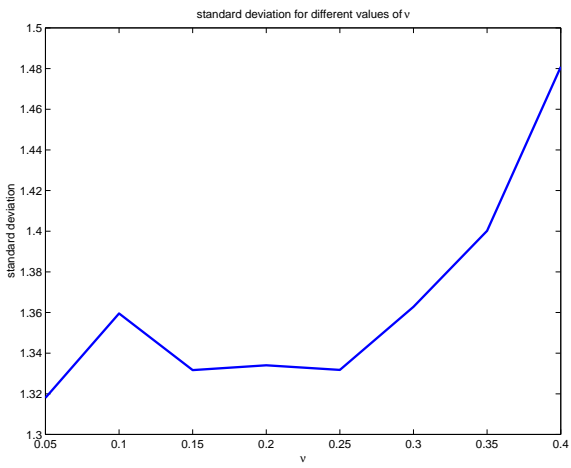

(b) Standard deviation of the errors for different values of $\nu$

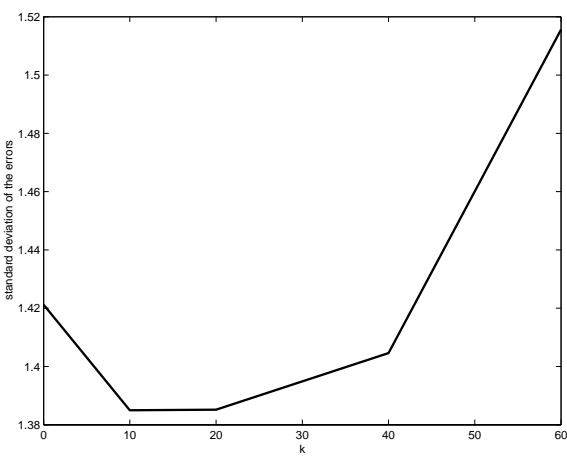

(d) Standard deviation of the errors for different values of $k$

Fig. 5. Mean and standard deviation of the error distribution over a patient's complete face of the predictions for different values of the Poisson parameter $\nu$ and growth parameter $k$.

Although the approximation is still not exact, it can immediately be seen that the prediction for the right cheek is much better in the extended model. This can also be seen in the histogram plot for the two error images (figure 7:a). The mean error $\mu=-0.57$ and the standard deviation $\sigma=1.43$ both have smaller values than for the basic model $(\mu=-1.05, \sigma=2.17)$ and thus make a better approximation of the Dirac pulse $\delta$.

As was indicated in the description of the validation procedure, the new method then needs to be validated on data of different patients, to make sure the results are also better in an arbitrary case. For this goal, the simulations were run with both the basic and the improved model on the data of 4 different patients. The error histograms compared to the real post-operative images were put together and averaged (figure $7 \mathrm{~b}$ ).

The results are less remarkable (because of the averaging operation), but it is still clear that the results with the improved model have errors (when compared 


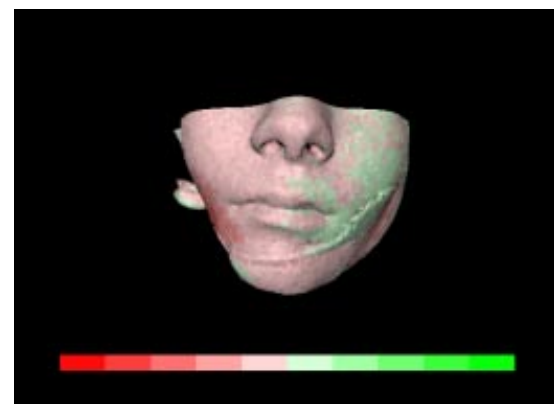

(a) Errors using the basic model

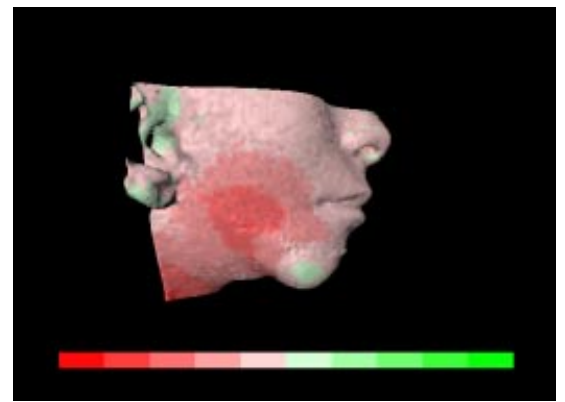

(c) Errors using the basic model

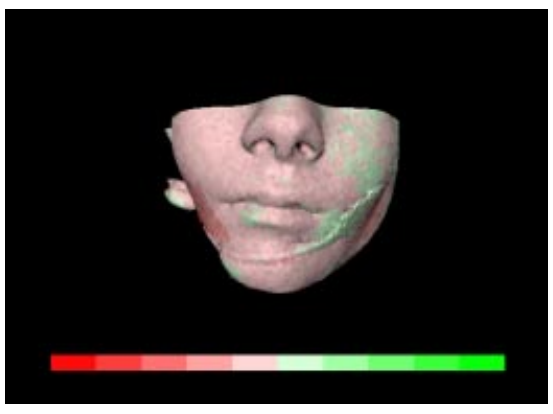

(b) Errors using the model including tissue growth $(\mathrm{k}=20)$

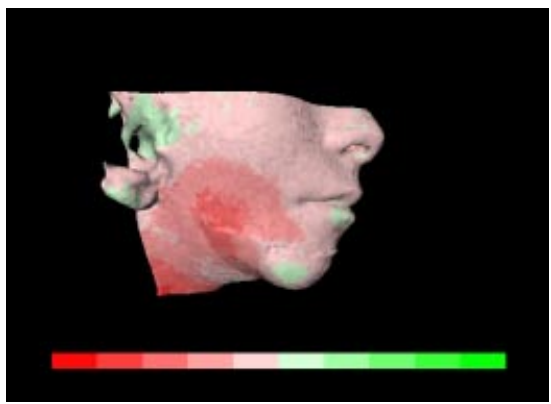

(d) Errors using the model including tissue growth $(\mathrm{k}=20)$

Fig. 6. Color-encoded differences between the real post-operative image and the planned image with the basic model (left) and with the extended model including tissue growth (right). The color scale covers the interval $[-10 \mathrm{~mm} \ldots 10 \mathrm{~mm}]$.

to the real post-operative images $)$ which are more centered around $0(\mu=-0.06$ compared to $\mu=-0.55)$ and have a smaller standard deviation $(\sigma=2.15$ compared to $\sigma=2.66$ ) than when the basic model is used.

\section{Discussion}

An important goal of maxillofacial surgery planning is to give the surgeon and the patient an accurate idea about what the face will look like as a result of the surgery. People are interested in a prediction of the facial outlook when swelling etc. has gone. They want to know what the patient will look like a few months after surgery, on the long term.

Therefore we need to incorporate long-term tissue behavior - like tissue growth - into the model used. Because of the large stresses induced by surgery, soft tissue grows considerably and has a large effect on the facial skin surface.

The validation results show the improvements made to the soft tissue model by including tissue growth. This definitely increases the accuracy by which maxillofacial surgery can be modelled. On the other hand, the validation also revealed 


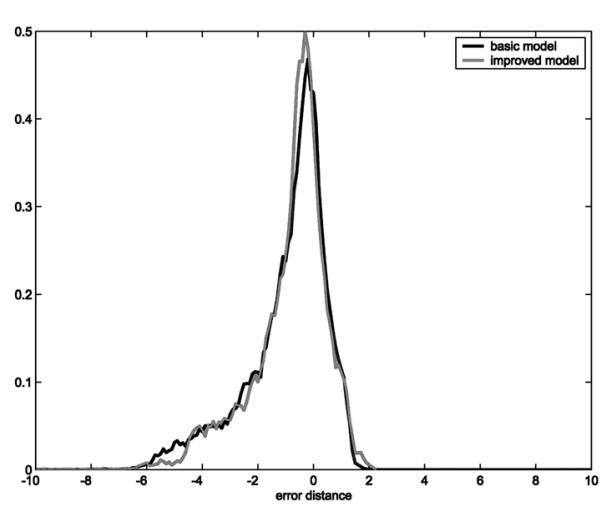

(a) One patient

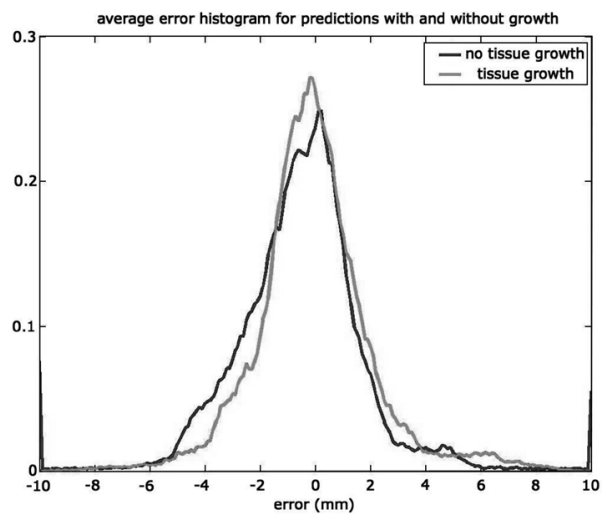

(b) Average of 4 patients

Fig. 7. Histogram data, comparison between the basic model and the extended model (growth parameter $k=20$ ).

that there are still relatively large areas in which the skin surface is modelled incorrectly.

The type of improvements to be made in an next step is not a simple question. Most people can easily say whether two faces are different (even with small differences) or not, but have difficulties to point the differences. In a similar way we see that our models are still not precise enough but it is difficult to find the refinements needed to make this last step.

Intuitively a first refinement could be made by discriminating muscles and fat as different soft tissue types with different characteristics. A next challenge will then certainly be to determine the characteristics of those different soft tissue types, as they are still largely unknown.

The boundary conditions form another field where important improvements could be made. It would be very useful to know precisely if the soft tissue next to the displaced bone elements stays fixed to it, or what happens exactly. And which parts of the soft tissue do not deform given a certain type of osteotomy? These are all questions which are still unanswered, but which can dramatically influence the quest for facial soft tissue models.

\section{Conclusion}

We developed a new soft tissue deformation prediction model for maxillofacial surgery planning. Starting from a simple, linear elastic 3-dimensional finite element model, a new model was constructed which includes the soft tissue growth during the months after surgery. An extensive validation procedure was also developed, which enabled us to objectively compare results with different methods.

The results obtained with this new model including tissue growth show a significant improvement over the results without tissue growth. The planned post-operative images match the real post-operative images much better now, 
making the surgery planning more useful to surgeons and patients. But before they can be really applied in practice, some further refinements still need to be made, as proposed in the discussion.

\section{Acknowledgments}

The work discussed here belongs to a grant for research specialization from the Flemish Institute for stimulation of the scientific-technological research in the industry (IWT) to Filip Schutyser. This work also partly belongs to the Flemish government IWT GBOU 020195 project on Realistic Image-based Facial Modeling for Forensic Reconstruction and Surgery Simulation.

\section{References}

1. F. Schutyser, J. Van Cleynenbreugel, M. Ferrant, J. Schoenaers, P. Suetens: Imagebased 3D planning of maxillofacial distraction procedures including soft tissue implications. Proceedings 3rd international conference on medical image computing and computer-assisted intervention - MICCAI2000, lecture notes in computer science, vol. 1935, pp. 999-1007, October 11-14, 2000

2. Y.C. Fung: Biomechanics: Mechanical properties of Living Tissues. 2nd edition. Ch. 7, Springer-Verlag, 1993, p. 242-320

3. M. Teschner, S. Girod, B. Girod: Optimization Approaches for Soft-Tissue Prediction in Craniofacial Surgery Simulation. Proceedings 2nd international conference on medical image computing and computer-assisted intervention - MICCAI'99, lecture notes in computer science, vol. 1679, p. 1183-1190, September 19-22, 1999

4. K. Waters: A physical model of facial tissue and muscle articulation derived from computer tomography data. SPIE Vol. 1808, Visualization in Biomedical Computing, volume 21, p. 574-583, 1992.

5. R.M. Koch, M.H. Gross, F.R. Carls, D.F. von Büren, G. Fankhauser, Y. Parish: Simulating Facial Surgery Using Finite Element Methods. SIGGRAPH 96 Conference Proceedings, 1996, p. 421-428

6. M. Chabanas, Y. Payan: A 3D Finite Element Model of the Face for Simulation in Plastic and Maxillo-Facial Surgery. Proceedings of MICCAI 2000, pp. 1068-1075

7. E. Gladilin, S. Zachow, P. Deuflhard, H.-C. Hege: Adaptive Nonlinear Elastic FEM for Realistic Soft Tissue Prediction in Craniofacial Surgery Simulations, SPIE Medical Imaging, San Diego, 2002.

8. O.C. Zienkiewicz, R.L. Taylor: The finite element method. Part 1: The basis, Butterworth Heinemann Oxford, 2000

9. F. Maes, A. Collignon, D. Vandermeulen, G. Marchal, P. Suetens: Multimodality image registration by maximization of mutual information, IEEE Transactions on Biomedical Imaging, April 1997, Vol. 16:2, pp 187-198

10. W. E. Lorensen, H.E. Cline: Marching Cubes: a High Resolution 3D Surface Construction Algorithm, ACM Computer Graphics \& Applications, November 1991, Vol. 11:6, pp 53-62

11. E. K. Rodriguez, A. Hoger, A. D. McCulloch: Stress-dependent finite growth in soft elastic tissues, Journal of Biomechanics, 1994, Vol. 27:4, pp 455-467

12. S. Balay, K. Buschelman, W. D. Gropp, D. Kaushik, L. C. McInnes, B. F. Smith: PETSc 2.0 Portable Extensible Toolkit for Scientific Computations, http://www.mcs.anl.gov/petsc, 2001 